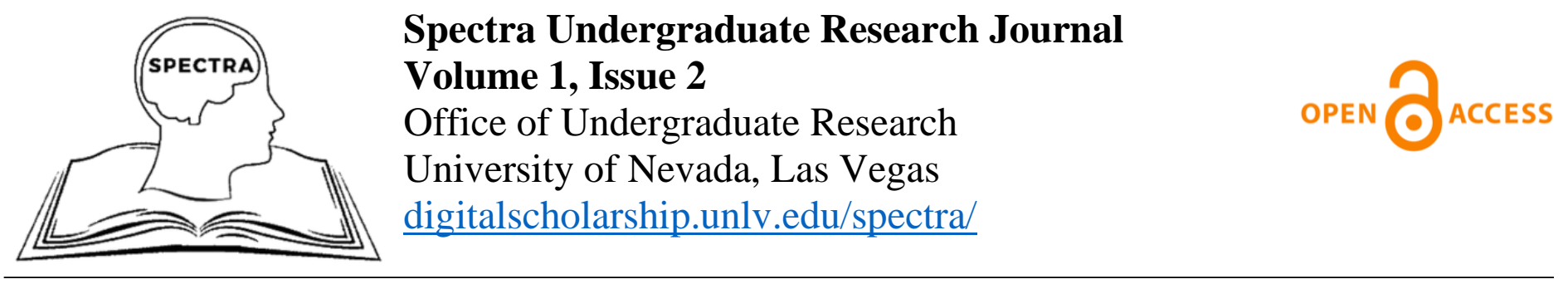

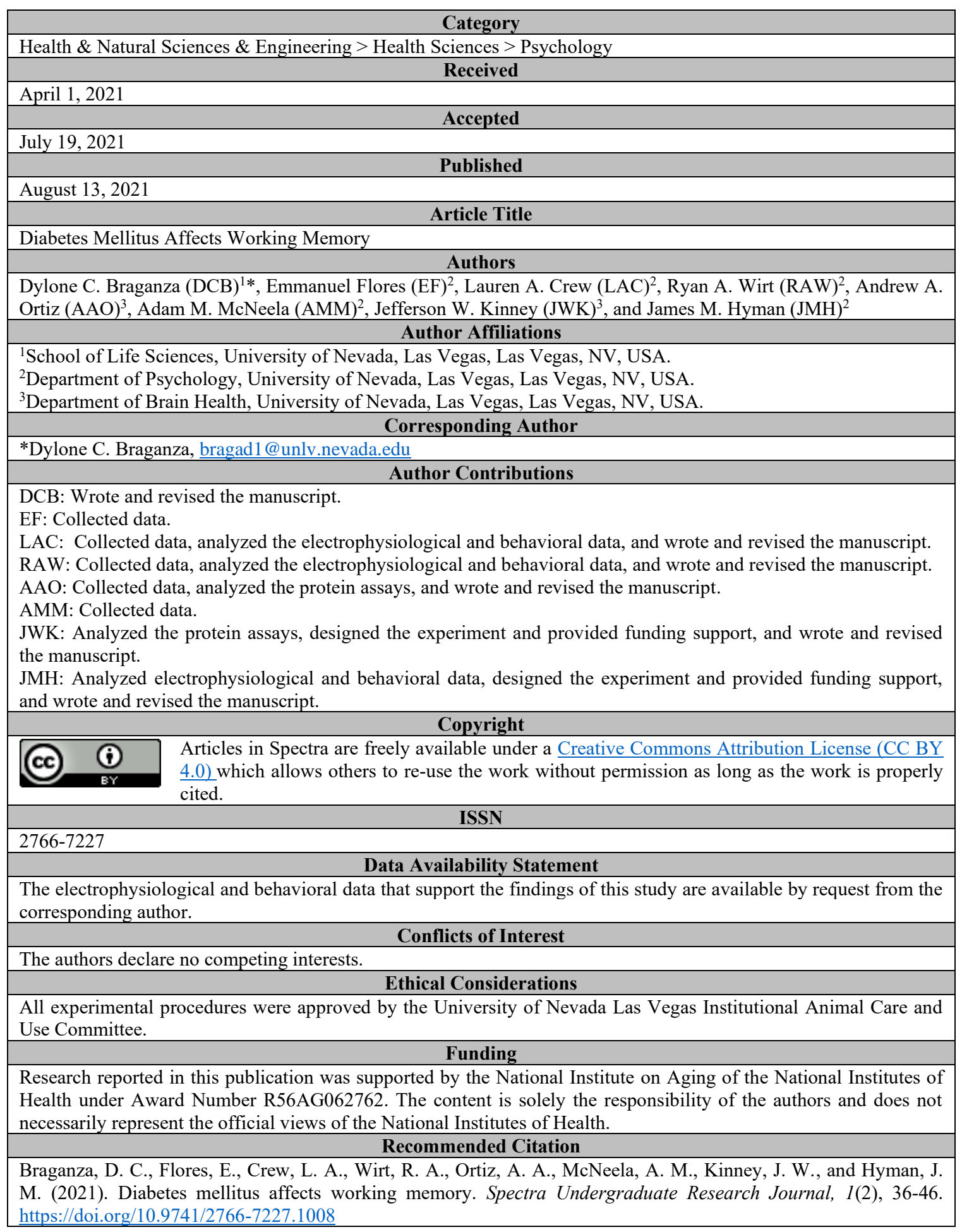




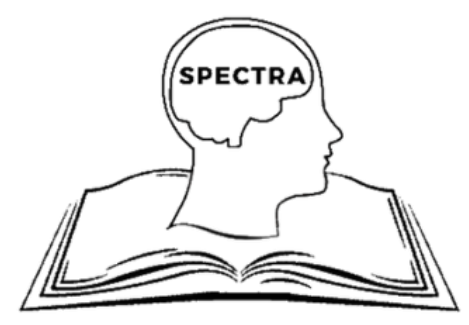

Spectra Undergraduate Research Journal

Volume 1, Issue 2

Office of Undergraduate Research

University of Nevada, Las Vegas

digitalscholarship.unlv.edu/spectra/

\title{
Diabetes Mellitus Affects Working Memory
}

Dylone C. Braganza ${ }^{1}$, Emmanuel Flores ${ }^{2}$, Lauren Crew ${ }^{2}$, Ryan Wirt ${ }^{2}$, Andrew A. Ortiz ${ }^{3}$, Adam M. McNeela ${ }^{2}$, Jefferson W. Kinney ${ }^{3}$, and James M. Hyman ${ }^{2}$

\section{Author Affiliations:}

${ }^{1}$ School of Life Sciences, University of Nevada, Las Vegas, Las Vegas, NV, USA.

${ }^{2}$ Department of Psychology, University of Nevada, Las Vegas, Las Vegas, NV, USA.

${ }^{3}$ Department of Brain Health, University of Nevada, Las Vegas, Las Vegas, NV, USA.

\begin{abstract}
Alzheimer's disease (AD) degrades the brain's ability to remember, think, and carry out tasks. The exact cause is not known, but several risk factors have been identified, including diabetes mellitus (DM). DM causes elevated blood sugar levels due to reduced insulin production in the pancreas. The linkage between elevated glucose levels and the behavioral impairments are not fully understood, which was the focus of this study. Rats were trained to alternate directions in a maze to receive a reward on consecutive trials. After training, five rats were injected with streptozotocin (STZ), which induces hyperglycemia by injuring pancreatic beta cells. Three control animals received benign vehicle injections. All eight rats then underwent implant surgery and received an implant with 128 recording probes attached to an electronic interface board. The recording electrodes targeted the hippocampus and the anterior cingulate cortex (ACC), which are both associated with learning and memory processes. We found that STZ rats had reduced accuracy after long delay periods compared to the control rats. During task performance, there was a decrease in the power of theta activity and an increase in delta activity moments before starting a new trial. This was the opposite of control animals, who before starting new trials had higher theta power and less delta power as they focused. These findings imply that the STZ rats were impaired on longer delay periods. These findings are like reports from animal models of AD and may help $\begin{array}{lllllll}\text { explain why } & \text { a } & \text { is } & \text { fisk } & \text { for } & \text { AD. }\end{array}$
\end{abstract}

Keywords: Alzheimer's disease, diabetes mellitus, streptozotocin, learning, memory, working memory, anterior cingulate cortex, hippocampus

Diabetes mellitus (DM) and Alzheimer's disease (AD) are prevalent diseases within our population that affect millions of people worldwide and can serve to be quite deadly. In 2013, the sixth leading cause of death in the United States was AD (Alzheimer's Association) and DM was the eighth leading cause of death (Tao et al., 2015). AD is a form of dementia that is most prevalent in elderly populations and is categorized by deficits in cortical function, specifically memory (Becker et. al, 1994). $\mathrm{DM}$ is categorized by elevated blood glucose levels, also known as hyperglycemia, and can occur due to dysfunction of insulin production or secretion (American Diabetes Association, 2014). Recently, it has been shown that DM is likely to be a risk factor for the development of Alzheimer's disease ( $\underline{\mathrm{Li}}$, Wang, \& Xiao, 2016). This is problematic since AD does not present the full extent of its symptoms until much later into the disease, and by this stage of the disease there are very few effective treatments known today. Both diseases fall within the top ten leading causes of mortality and understanding comorbidity 
interactions can uncover how memory, specifically working memory, is affected by hyperglycemic states. The focus of this study was placed on changes in working memory in hyperglycemic rodents. To investigate any changes in working memory, brain structures tasked with the consolidation and retrieval of memory were focused on.

Two brain areas that have shown to be integral to understanding the underlying pathologies of memory from DM are the anterior cingulate cortex (ACC) and the hippocampus (HC). The ACC is active in relation to emotional behavior and decision-making regarding rewards (Rolls, 2019). The HC is also known to be involved in declarative, episodic, (Opitz, 2014), and spatial memory (Schlesiger et. al, 2013). In rodents, ablation to the hippocampal area removes the ability to complete a delayed alternation task (Racine \& Kimble, 1965). Also, previous studies have shown that treating hyperglycemia in humans can improve cognition and working memory test scores (Ryan et. al, 2006).

In our model organisms, DM is induced with the neoplastic agent streptozotocin (STZ), and it is known to induce an elevated blood glucose or DMlike state in rodents (Murtishaw et. al, 2018). Hyperglycemic states have been implicated in leading to cognitive decline in relation to memory function, even when the individual does not meet the threshold for a clinical diagnosis of type 2 diabetes (Marden et. al, 2017). Harvesting information from brain areas that are implicated in working memory and decision making when blood glucose levels are elevated will allow the advancement of future interventions that will hopefully treat and improve the status of DM.

By understanding the effect on working memory by DM, a clearer picture of the cognitive implications of DM can be uncovered. Furthermore, measuring behavior modulations in hyperglycemic rodents can identify changes to the function of working memory. This distinction will help identify the relationship between the hallmark symptoms that define DM, which is hyperglycemia, and correlate any dementia-like symptoms with them.

\section{Methodology}

Eight Long-Evans rats were trained on a delayed alternation task in a custom-built T-maze. For the rats to have completed successful trials in succession, they would have had to travel left then right within the T-maze, and then repeat for their reward. The maze featured a sequester area where the rats would be forced to wait for a variable amount of time before proceeding onto the next trial. This required the animals to maintain the memory of the last trial over this delay period. When the rats were then released into the start area, they traveled some distance and encountered two separate pathways. At the end of one pathway, a reward was waiting for the rats while the other path contained no reward. This pattern alternated throughout consecutive trials.

Five out of the eight rats received STZ injections, and three rats received a benign vehicle injections that contained a citrate buffer following training to ensure that the rodents could correctly complete the task. All injections were delivered intraperitoneally. The STZ animals exhibited average glucose levels of $358 \mathrm{ng} / \mathrm{ml}$, with the threshold of 250 $\mathrm{ng} / \mathrm{ml}$ for a chronic state of hyperglycemia, commonly found in DM patients (Murtishaw et al., 2018). Following surgery and adequate training in the T-maze, all of the rats underwent surgery for the implantation of a 128 channel hyperdrive recording probe. The implant surgery targeted the ACC as well as the $\mathrm{HC}$, specifically the CA1 region. Wires were arranged in groups of four, which are known as tetrodes. The tetrodes were attached to an electronic interface board to properly record brain activity during the tasks. During surgery, the tetrodes were lowered into the HC and the ACC. All eight rats ran the task again while activity from both brain areas was recorded. Following recordings from the animal's brain areas, the animals were sacrificed, and histological inspection confirmed that the electrodes were implanted in the target areas.

Delta and theta waves are brain oscillations that are used to correlate brain activity with behavior. Theta waves are present during a task that involves active attention and focus (Buzsáki et. al, 1986), and are found between 7-12 Hz. Delta waves are correlated with resting states and are found between 1-4 Hz (Fujisawa \& Buzsáki, 2011). Figure 2 shows brackets denoting a period of delta (orange) and theta (purple) waves. 


\section{Results}

Task Accuracy: The accuracy for STZ animals diminished after delay periods longer than 60 seconds and above compared to the control group (Figure 3). Within trials with delay periods of 60 seconds or less, both groups had similar accuracies of correct trials. This pattern persisted into the 60 second delay period with similar accuracies for STZ and control rats. Upon encountering a 90 second delay period, accuracy for STZ rats plummeted to $60 \%$ and plateaued at this accuracy level. The control group responded correctly to more than $10 \%$ of the trials compared to the STZ rats. In trials consisting of a delay period above 90 seconds, both groups responded with a similar accuracy.

Differences in Delta and Theta Waves: Power differences in theta and delta waves within the HC were apparent between the two groups. Starting with the control group (left), the red band at $\sim 7 \mathrm{~Hz}$ shows a constant theta power while the subjects waited for the trial start (Figure 4). Comparing these results to the STZ group, an increase in delta power $(1-4 \mathrm{~Hz})$ can be seen throughout the trial, which is not observed in the control group. Notice that there is a consistency of power throughout the time leading up to the trial start, denoted by the vertical dashed line, as well as a few seconds following in the control group. A slight fluttering of increased delta power can be seen leading up to the trial start in the STZ animals, which is absent within the control group.

TD Ratio Shows Behavioral Difference: To parse out the amount of time that the animals spent in each type of behavioral condition, whether it was attentive or lethargic, a theta to delta (TD) ratio was calculated. The TD ratio will give an estimate of the animal's behavior due to the correspondence between the specific waves and behavior. When comparing the TD ratio before the trial start within the control and experimental groups, the control groups persisted at a higher ratio (Figure 5). A higher TD ratio signifies that there was more theta activity than delta activity present while the animal was waiting the task to begin. The TD ratio of the experimental group was lower than the control group, which suggests that the STZ group was unable to focus and pursue the reward at the level that the control group was able to. Both groups showed an increase in TD ratio leading up to the trial start.
Both groups received equal amounts of time for the trials in the experiment (Figure 6a). This was to ensure that every animal had equal opportunities to wait within the sequester area, as well as to keep the variable of delay time constant. The experimental and control groups remained at a constant weight for the first two weeks but differed in the amount of weight after 4 weeks of the vehicle injections, with the STZ group losing more weight compared to the control group (Figure 6b).

\section{Discussion}

These results show that elevated blood glucose levels have the capability to affect working memory during longer delay periods. Shorter delays were not as impaired in the hyperglycemic subjects, possibly implicating a disconnect between DM conditions and working memory. The memory deficits that preceded the hyperglycemic state mirror conditions seen in AD animal models and AD patients (Stopford et. al, 2012). Even though the effects of hyperglycemia and working memory have been identified, much is still unknown about $\mathrm{DM}$ and $\mathrm{AD}$. One connection between both diseases is the necessity of healthy blood glucose levels, as hypoglycemic states can also cause cognitive impairments (Sommerfield et. al, $\underline{2003}$ ).

Future studies that are looking to peer into hyperglycemia and its cognitive effects could also perform operations that investigate various pairwise combinations of other brain areas that are involved in learning and memory. These future areas should be highly integrated with either the hippocampus or ACC, like the orbitofrontal cortex (Rolls, 2019), which would require other mechanisms of investigation other than electrophysiology to parse out directly. One could also perturb one brain area and leave another intact, allowing for multiple pairwise combinations of impaired and non-impaired cortical regions. Aside from learning and memory impairments that are present with any patient with $\mathrm{AD}$, other cognitive functions that are impaired could be investigated by examining other cortical areas that are associated with emotional arousal, like the amygdala. These findings also aid in furthering our focus on advanced glycosylation end products, which are known to affect cellular functions by and large, but the role of glycosylation in neural pathology is not 
fully unraveled yet. By working from the molecular level up to the cognitive level, one could build upon how cellular processes involving glucose affect memory.

\section{References}

Alzheimer's Association (2016). 2016 Alzheimer's disease facts and figures. Alzheimer's \& Dementia, 12. 459-509.

American Diabetes Association (2014). Diagnosis and classification of diabetes mellitus. Diabetes care, 37 Suppl 1, S81-S90.

https://doi.org/10.2337/dc14-S081

Becker, J.T., Boiler, F., Lopez, O.L., Saxton, J., McGonigle, K.L. (1994). The Natural History of Alzheimer's Disease: Description of Study Cohort and Accuracy of Diagnosis. Arch Neurology, 51, 585-594. doi:10.1001/archneur.1994.00540180063015

Buzsáki, G., Gage, F., Björklund, A. (1986). Restoration of RSA (theta) in the Denervated Hippocampus by Brain Transplants. Learning and Memory, 137-140. https://doi.org/10.1016/B978-0-08-0341866.50027-X

Fujisawa, S., Buzsáki, G. (2011). A 4 Hz Oscillation Adaptively Synchronizes Prefrontal, VTA, and Hippocampal Activities. Neuron, 72, 153165. https://doi.org/10.1016/j.neuron.2011.08.018

Li, W., Wang, T., \& Xiao, S. (2016). Type 2 diabetes mellitus might be a risk factor for mild cognitive impairment progressing to Alzheimer's disease. Neuropsychiatric Disease and Treatment, 12. https://doiorg.ezproxy.library.unlv.edu/10.2147/NDT.S1 11298

Marden, J., Mayeda, E., Tchetgen, E., Kawachi, I., Glymour, M. (2017). High Hemoglobin A1c and Diabetes Predict Memory Decline in the Health and Retirement Study. Alzheimer Disease \& Associated Disorders, 31, 48-54. https://doi.org/10.1097/WAD.0000000000000 182

Murtishaw, A. S., Heaney, C. F., Bolton, M. M., Belmonte, K. C. D., Langhardt, M. A., \& Kinney, J. W. (2018). Intermittent streptozotocin administration induces behavioral and pathological features relevant to Alzheimer's disease and vascular dementia. Neuropharmacology, 137, 164-177. https://doiorg.ezproxy.library.unlv.edu/10.1016/j.neurop harm.2018.04.021

Opitz, B. (2014). Memory function and the Hippocampus. Frontiers of Neurology and Neuroscience, 34. https://doi.org/10.1159/000356422

Racine, R., Kimble, D. (1965). Hippocampal lesions and delayed alternation in the rat. Psychonomic Science, 3. 285-286. http://dx.doi.org/10.3758/BF03343139

Rolls E. T. (2019). The cingulate cortex and limbic systems for emotion, action, and memory.

Brain structure \& function, 224(9), 30013018. https://doi.org/10.1007/s00429-01901945-2

Ryan, C., Freed, M., Rood, J.A., Cobitz, A.R., Waterhouse, B.R., Strachan, M. (2006). Improving Metabolic Control Leads to Better Working Memory in Adults with Type 2 Diabetes. Diabetes Care, 29. 345-351. https://doi.org/10.2337/diacare.29.02.06.dc051626

Schlesiger, M., Cressey, J.C., Boublil, B., Joenig, J., Melvin, N., Leutgeb, J.K., Leutgeb, S. (2013). Hippocampal activation during the recall of remote spatial memories in radial maze tasks. Neurobiology of Learning and Memory, 106, 324-333. https://doi.org/10.1016/j.nlm.2013.05.007

Sommerfield, A.J., Deary, I.J., McAulay, V., Frier, B.M. (2003). Short-Term, Delayed, and Working Memory are Impaired During Hypoglycemia in Individuals with Type 1 Diabetes. Diabetes Care, 26. 390-396. https://doi.org/10.2337/diacare.26.2.390

Stopford, C.L., Thompson, J.C., Neary, D., Richardson, A.M.T., Snowden, J.S. (2012). Working memory, attention, and executive function in Alzheimer's disease and frontotemporal dementia. Cortex, 48. 429-446. https://doi.org/10.1016/j.cortex.2010.12.002

Tao, Z., Shi, A., \& Zhao, J. (2015). Epidemiological 
Perspectives of Diabetes. Cell biochemistry https://doi.org/10.1007/s12013-015-0598-4 and biophysics, 73(1), 181-185. 
Appendix A: Figure 1. T-maze configuration and BGI analysis

A

Delayed Alternation Task

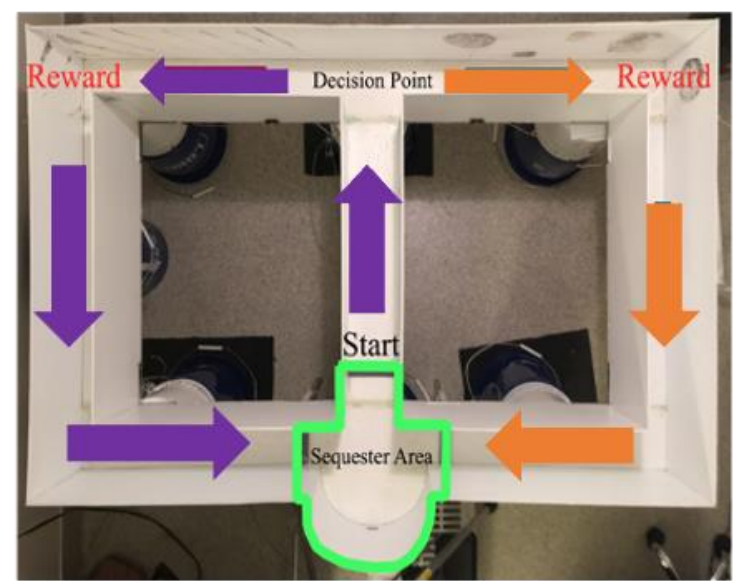

B

Blood Glucose Index

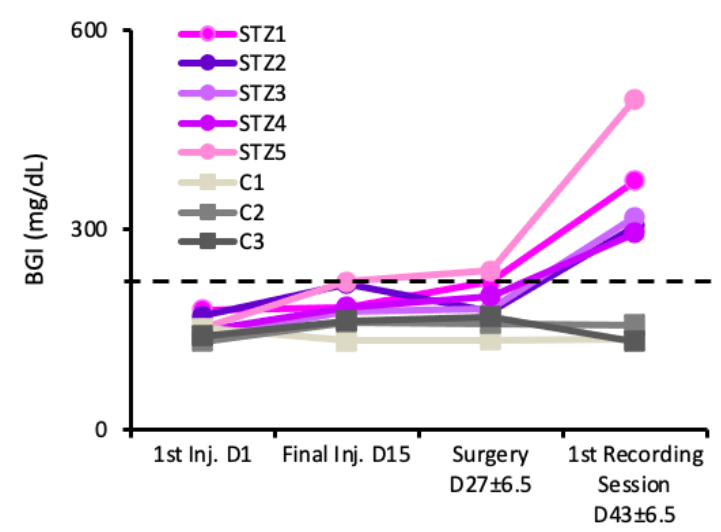

Note. Figure 1a: Custom built T-maze (left) shows the sequester area outlined in green, which was where the rodents waited for the trial to start. Both available pathways (purple and orange arrows) and reward areas are also shown. Figure 1b: Blood glucose levels were measured using a blood glucose index (BGI). Levels of BGI was measured and tracked through the time from the first injection of STZ to the time of the first recording session (right). All STZ animals were hyperglycemic at the time of the first recording session. 
Appendix B: Figure 2. Delta and theta wave example

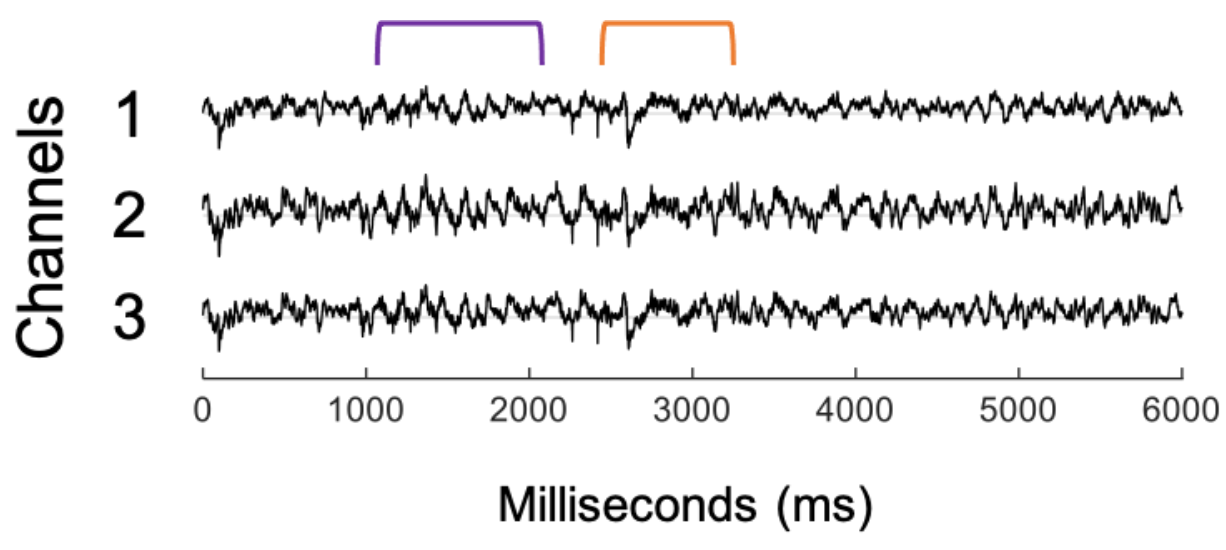

Note. An example of electrical activity during a 6 second recording session through an EEG. The y-axis shows three separate EEG channels, and the $\mathrm{x}$-axis denotes the time passed in milliseconds. The oscillating waves during the 1000-2000 ms period is an example of theta waves (purple), and delta waves are shown between the 2500-3200 ms period (orange). 
Appendix C: Figure 3. Task accuracy analysis

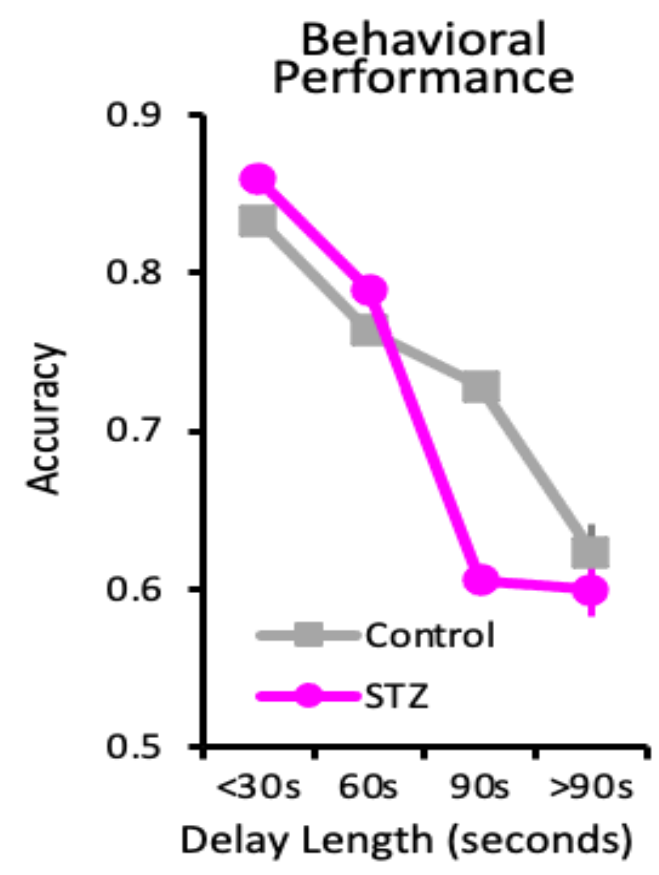

Note. Differences in behavioral performance between groups is shown by comparing different delay periods with the percentage of correct trials completed. The accuracy of the correctly completed trials is on the y-axis and delay length in seconds is on the x-axis. Accuracy of the STZ group decreased after more than a 60 second delay period. 

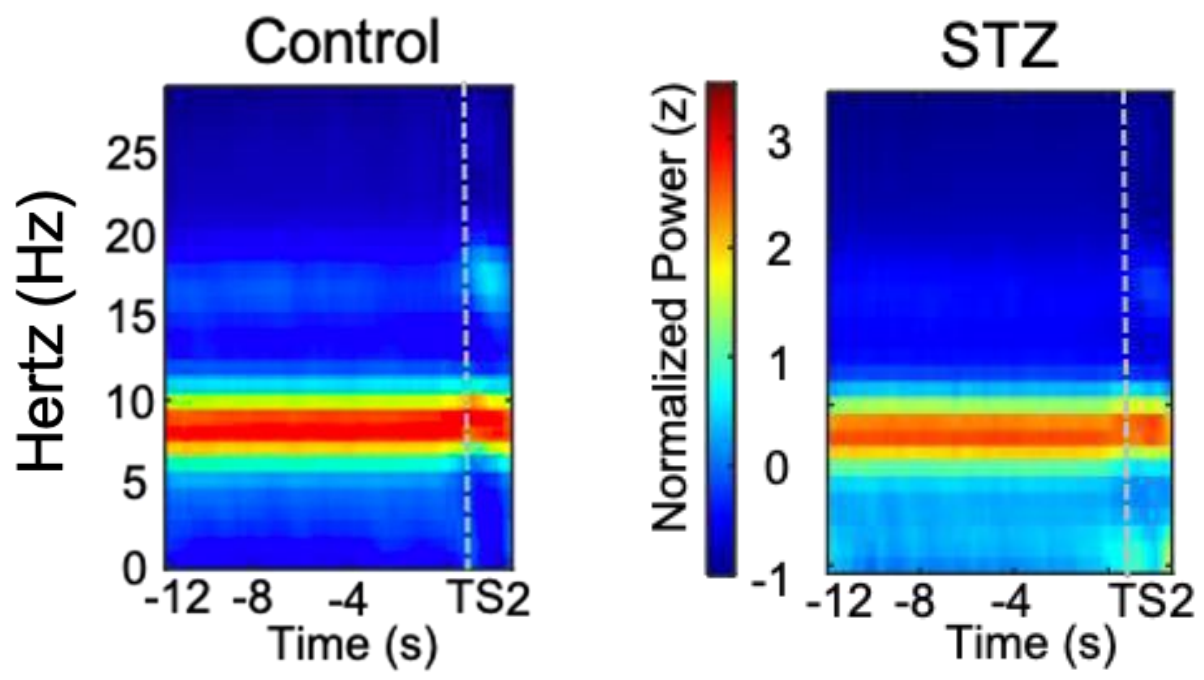

Note. Normalized power throughout the trial leading up to the trial start. Theta waves $(\sim 7-14 \mathrm{~Hz})$ and delta waves $(\sim 1-4 \mathrm{~Hz})$ are present in both graphs with different power. Frequency $(\mathrm{Hz})$ is displayed on the y-axis, and time leading up to the trial start (TS) is on the $\mathrm{x}$-axis. A dashed vertical line in each graph denotes the start of the trial. Normalized power (z) scale is shown in a blue to red gradient from -1 (low power) to 3 (high power). The STZ group showed a decrease in delta wave power compared to the control group leading up to the trial start, as well as a decrease in theta power. 


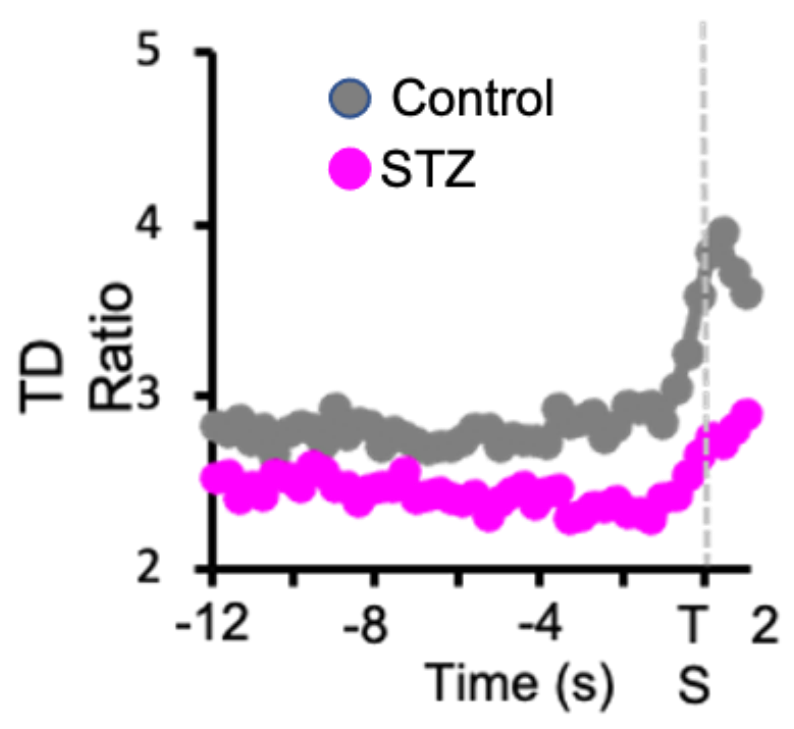

Note. Normalized power throughout the trial leading up to the trial start. Theta waves $(\sim 7-14 \mathrm{~Hz})$ and delta waves ( 1-4 Hz) are present in both graphs with different power. Frequency $(\mathrm{Hz})$ is displayed on the y-axis, and time leading up to the trial start (TS) is on the x-axis. A dashed vertical line in each graph denotes the start of the trial. Normalized power $(\mathrm{z})$ scale is shown in a blue to red gradient from -1 (low power) to 3 (high power). The STZ group showed a decrease in delta wave power compared to the control group leading up to the trial start, as well as a decrease in theta power. 
Appendix F: Figure 6. Body weight and delay length comparisons

A

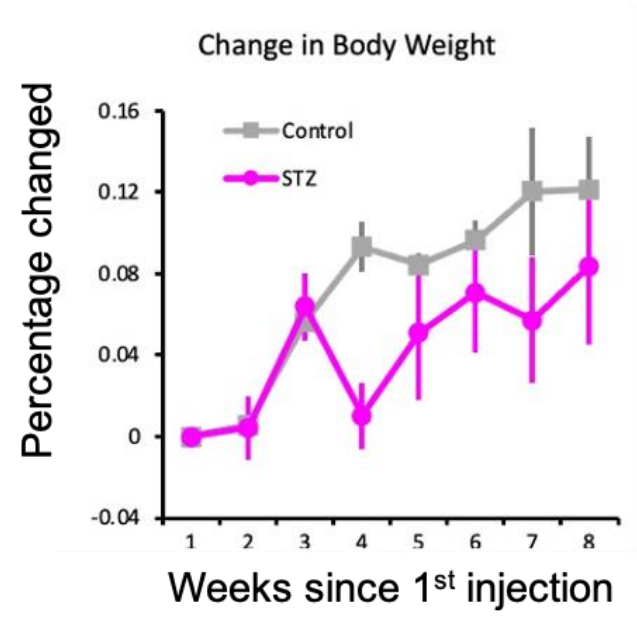

B

\section{Total Delay
Length}
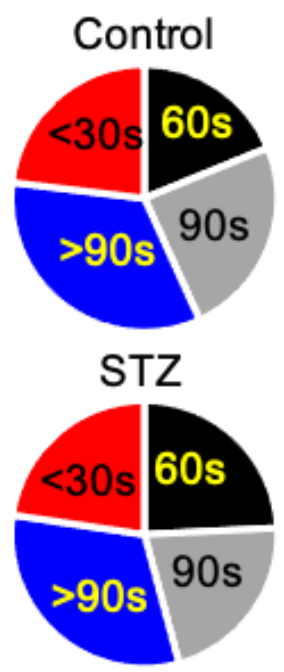

Note. Figure 6a: The percentage of change in body weight is shown throughout the weeks following the vehicle injections (STZ group) and citrate buffer (control group). Both groups increased in body weight throughout the first eight weeks (left). Figure 6b: All animals from both groups received the same amount of time within each delay period (right). 